\title{
Enhancement of External Damping of a Flexible Rotor in Active Magnetic Bearings by Time-Periodic Stiffness Variation*
}

\author{
Fadi DOHNAL** and Richard MARKERT** \\ ** Institute for Structural Dynamics, Technische Universität Darmstadt \\ Petersenstrasse 30, 64287 Darmstadt, Germany \\ E-mail: dohnal@sdy.tu-darmstadt.de
}

\begin{abstract}
Previous theoretical studies have shown analytically and numerically that a vibrating system can be stabilised and its vibrations can be suppressed by an open-loop control of a stiffness parameter, a stabilisation by parametric stiffness excitation. This approach is investigated further numerically and implemented experimentally for a flexible rotor with multiple disks supported by active bearings. A periodic open-loop control of the stiffness coefficients of a bearing is realised by periodically changing the control parameters of an active magnetic bearing. This periodic variation of control parameters is regulated at fixed frequency and amplitude in such a way that it acts like a parametric excitation in the rotor system. As it was shown for simple vibrating structures (chain mass system, cantilever, Jeffcott rotor), a periodic variation can enhance the effective damping which leads to a vibration reduction in a vibrating system. Since this control is open-loop, it can be operated in parallel to existing and well-established controllers already in use in active magnetic bearings.

In this paper, the method of damping by parametric excitation is realised experimentally in a rotor system. Direct numerical simulation is performed to calculate ranges for control and system parameters where damping by parametric excitation is effective. First experimental results are shown to demonstrate the applicability of the method.
\end{abstract}

Key words : Flexible Rotor, Active Magnetic Bearings, Parametric Excitation

\section{Introduction}

Systems of differential equations with periodic coefficients, also termed parametrically excited systems, have been the focus of scientific research for a long time. Parametrically excited vibrations may occur if one or more coefficients of the differential equations of motion are not constant but periodically time-varying. The frequency of the parameter change is prescribed explicitly as a function of time and is independent of the motion of the system. Classical examples are a pendulum with a periodically varying length or a periodically moving pivot point or a rotating shaft with a nonsymmetric cross-section. Parametrically excited systems and structures have been studied extensively in the past because of the somehow unexpected dynamic phenomena that occur in such systems. A parametrically excited system may exhibit a destabilising parametric resonance if the variation frequency is close to ${ }^{(11),(12)}$

$$
v_{0}=\frac{\left|\omega_{k} \mp \omega_{l}\right|}{n}, \quad k=1,2, \ldots
$$

Herein $\omega_{i}$ denotes the $i$ th natural frequency of the underlying undamped system and $n$ is a natural number. Several publications deal with a single or coupled differential equations having time-periodic coefficients ${ }^{(1),(2),(8)}$. The main focus there was to investigate the destabilising effect of parametric excitation, i.e. the instability boundary curves in the domain of system parameters. The non-resonant cases were not considered to be relevant for applications. The 
mechanism of damping by parametric excitation, as investigated here, is based on the coupling of vibration modes which leads to an artificial increase of the overall damping in the system. A specific control frequency at which the system vibrations are reduced is termed as a parametric anti-resonance frequency. The main theoretical contributions with respect to parametric anti-resonances in this context can be found in ${ }^{(3),(6),(9)}$.

Very few studies have been undertaken to verify the existence of parametric antiresonances experimentally. Mainly discrete 2DOF systems of an artificial nature have been investigated so far and a single study of a continuous structure, a cantilever with time-periodic stiffness support $^{(4)}$. The main motivation of the present study is to prove experimentally that the concept of damping by parametric excitation is applicable for damping the low-frequency modes of a complex, flexible rotor system.

A parametric excitation can be introduced in the system in a simple open-loop manner, since the stiffness parameter in focus needs to be varied at a well-defined, fixed frequency and fixed amplitude. The present paper examines this open-loop strategy to increase the effective damping of a rotor system based on preliminary theoretical investigations ${ }^{(5),(10)}$. Therein, it was shown theoretically that a periodic change in the bearing stiffness is capable of increasing the critical speed of a simple Jeffcott rotor under the influence of a destabilising self-excitation. Now, this approach is used to enhance the damping of an already stable system and realised experimentally for a complex flexible rotor system supported by active magnetic bearings.

In the first section, the model equations of a flexible rotor system with multiple rigid disks attached to its shaft are derived. A numerical prediction is given for the regions where damping by parametric excitation is effective. Finally, first experimental results are compared to these theoretical predictions.

\section{Lateral vibrations of a flexible rotor with multiple rigid disks}

In this section, the equations of motion are derived for a flexible rotor shaft with multiple rigid disks attached to its shaft under the action of unbalance and electromagnetic forces. First, the system matrices and force vectors are listed. Then, they are assembled to the resulting equations of motion.

The mechanical model of the rotor system under consideration is shown in Fig. 1. A slender, flexible rotor shaft is rotating at a constant rotational speed $\Omega$ provided by a driving motor. The shaft is assumed to be torsionally rigid and isotropic. Five rigid disks are attached to the shaft, three disks (D1, D2, D3) and two bearing studs (AMB1, AMB2). The disks are assumed to be unbalanced. The shaft is supported by two active magnetic bearings (AMB1, AMB2). The actual stud position is measured and fed back to a PID controller that drives the power amplifiers of the electromagnets in the AMBs. The total length of the shaft is $680 \mathrm{~mm}$.

\subsection{Continuous shaft with discrete disks}

The flexible, continuous shaft is discretised using finite beam elements. For a finite beam element of length $l_{i}$, bending stiffness $E I$, cross-section $A$ and density $\rho$, the inertia and

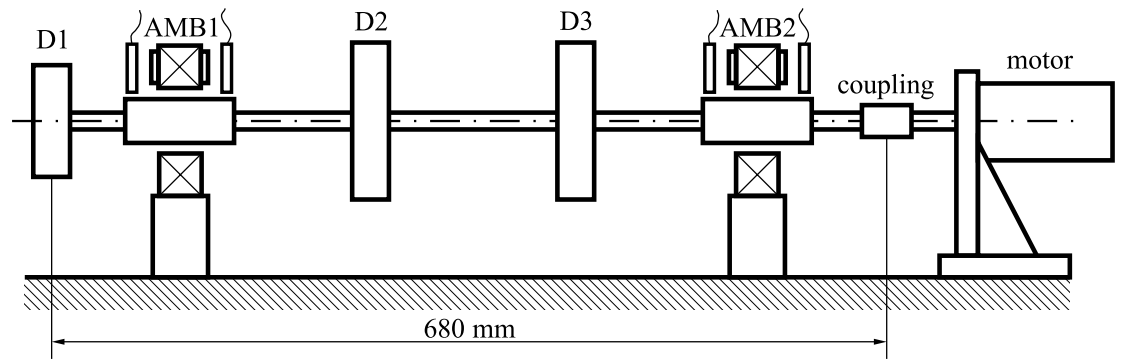

Fig. 1 Flexible rotor with multiple disks and electromagnetic supports. 
stiffness matrix become ${ }^{(7)}$

$$
\mathbf{M}_{b i}^{z / y}=\frac{\rho A l_{i}}{420}\left[\begin{array}{cccc}
156 & \mp 22 l_{i} & 54 & \pm 13 l_{i} \\
\mp 22 l_{i} & 4 l_{i}^{2} & \mp 13 l_{i} & -3 l_{i}^{2} \\
54 & \mp 13 l_{i} & 156 & \pm 22 l_{i} \\
\pm 13 l_{i} & -3 l_{i}^{2} & \pm 22 l_{i} & 4 l_{i}^{2}
\end{array}\right]
$$

and

$$
\mathbf{C}_{b i}^{z / y}=\frac{2 E I}{l_{i}^{3}}\left[\begin{array}{cccc}
6 & \mp 3 l_{i} & -6 & \mp 3 l_{i} \\
\mp 3 l_{i} & 2 l_{i}^{2} & \pm 3 l_{i} & l_{i}^{2} \\
-6 & \pm 3 l_{i} & 6 & \pm 3 l_{i} \\
\mp 3 l_{i} & l_{i}^{2} & \pm 3 l_{i} & 2 l_{i}^{2}
\end{array}\right]
$$

with respect to the element coordinate vector in $z$-direction and $y$-direction, respectively,

$$
\mathbf{q}_{i}^{z}=\left[\begin{array}{llll}
z_{i} & \varphi_{y i} & z_{i+1} & \varphi_{y i+1}
\end{array}\right]^{T}, \quad \mathbf{q}_{i}^{y}=\left[\begin{array}{cccc}
y_{i} & \varphi_{z i} & y_{i+1} & \varphi_{z i+1}
\end{array}\right]^{T} .
$$

Rigid disks of mass $m_{r}$ and moment of inertia $\Theta_{r}$ are attached at discrete positions along the shaft. Their symmetry axis is aligned with the central rotary axis. For rigid disks that are attached to the end points of a finite beam element, the corresponding mass and stiffness matrices have diagonal form and read

$$
\mathbf{M}_{r}=\left[\begin{array}{cccc}
m_{r i} & & & \\
& \Theta_{r i} & & \\
& & m_{r i+1} & \\
& & & \Theta_{r i+1}
\end{array}\right] .
$$

The element matrices in Eqs. (2), (3) and (5) are assembled to the global system matrices $\mathbf{M}_{b}^{z / y}, \mathbf{C}_{b}^{z / y}$ and $\mathbf{M}_{r}$ with respect to the global coordinate vectors

$$
\mathbf{q}^{z}=\left[\begin{array}{lllll}
z_{1} & \varphi_{y 1} & \cdots & z_{n} & \varphi_{y n}
\end{array}\right]^{T}, \quad \mathbf{q}^{y}=\left[\begin{array}{lllll}
y_{1} & \varphi_{z 1} & \cdots & y_{n} & \varphi_{z n}
\end{array}\right]^{T}
$$

\subsection{Active magnetic bearings}

Active magnetic bearings (AMBs) consist of pairs of electromagnets that are arranged, in general, in two perpendicular axes enabling to control the rotor position within a plane. Since electromagnets can apply only attracting but no repelling forces, two electromagnets are needed for each direction.

The electromagnetic force generated by the AMB depends on its geometry parameters (cross-section of the pole shoes, size of the air gap) and its electromagnetic properties (number of turns, permeability) and is a strongly nonlinear function of these parameters. In practice, however, the resulting force can be linearised close to a certain operation point as

$$
F_{m}^{l i n}=k_{i} i_{c}-k_{s} r
$$

assuming a high bias current for pre-magnetisation and small control current $i_{c}$ and rotor deflection $r$. Herein, $k_{i}$ is the current-force constant and $-k_{s}$ the negative bearing stiffness. Cross-coupling parameters are neglected.

The most widely used control concept for an AMB is a PID controller. The proportional $\left(k_{P}\right)$ and the derivative actions $\left(k_{D}\right)$ constitute the stiffness and damping characteristics of the bearing while the integral action $\left(k_{I}\right)$ assures that the resulting rotor deflection $r$ keeps track with a predefined setpoint,

$$
i_{c}=k_{P} r+k_{D} \dot{r}+k_{I} \int r d t .
$$

Inserting into Eq. (7) leads to

$$
F_{m}^{l i n}=c_{m} r+d_{m} \dot{r}+k_{i} k_{I} \int r d t
$$


with the active stiffness and active damping coefficients

$$
c_{m}=k_{i} k_{P}-k_{s}, \quad d_{m}=k_{i} k_{D} .
$$

Adjusting the control parameters $k_{P}$ and $k_{D}$ determines the dynamic properties of the AMB. Both AMBs are assumed to be isotropic. With the mechanical properties in Eq. (10), the stiffness and damping matrices with respect to the global coordinate vector have diagonal form with entries at the location of the AMBs (see Fig. 1),

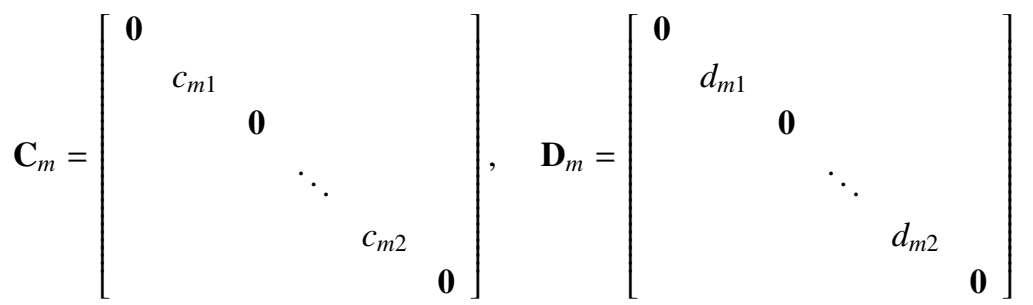

\subsection{Excitation forces}

The rotor system is excited by unbalance forces originating from eccentricities $\varepsilon_{i}$ of the five rigid disks of mass $m_{i}$ (including the bearing studs). Since the shaft is not bended, the unbalance force vectors can be written as

$$
\begin{aligned}
\mathbf{f}_{z} & =-(\cos \varphi(t))^{*}\left[\begin{array}{lllllll}
m_{1} \varepsilon_{1} & 0 & m_{2} \varepsilon_{1} & 0 & \ldots & m_{5} \varepsilon_{5} & 0
\end{array}\right]^{T}, \\
\mathbf{f}_{y} & =-(\sin \varphi(t))^{*}\left[\begin{array}{lllllll}
m_{1} \varepsilon_{1} & 0 & m_{2} \varepsilon_{1} & 0 & \ldots & m_{5} \varepsilon_{5} & 0
\end{array}\right]^{T},
\end{aligned}
$$

where $\varphi(t)$ is the rotary angle of the shaft.

\subsection{Equations of motion}

The element matrices from Eqs. (2), (3) and (5) are assembled to global system matrices with respect to the global coordinate vectors in Eq. (6). Together with the global system matrices in Eq. (11) describing the electromagnetic actions and the unbalance forces in Eq. (12), the equations of motion of the rotor system with respect to the global coordinate vector $\mathbf{q}=\left[\mathbf{q}^{z, T}, \mathbf{q}^{y, T}\right]^{T}$ become

$$
\mathbf{M} \ddot{\mathbf{q}}+\mathbf{D} \dot{\mathbf{q}}+\mathbf{C q}=\mathbf{f}
$$

with the assembled coefficient matrices and the global force vector

$$
\begin{aligned}
& \mathbf{M}=\left[\begin{array}{cc}
\mathbf{M}_{b}^{z}+\mathbf{M}_{r} & \mathbf{0} \\
\mathbf{0} & \mathbf{M}_{b}^{y}+\mathbf{M}_{r}
\end{array}\right], \quad \mathbf{D}=\left[\begin{array}{cc}
\mathbf{D}_{m} & -\Omega_{\mathbf{G}_{r}} \\
\Omega \mathbf{G}_{r} & \mathbf{D}_{m}
\end{array}\right], \\
& \mathbf{C}=\left[\begin{array}{cc}
\mathbf{C}_{b}^{z}+\mathbf{C}_{m} & \mathbf{0} \\
\mathbf{0} & \mathbf{C}_{b}^{y}+\mathbf{C}_{m}
\end{array}\right], \quad \mathbf{f}=\left[\begin{array}{l}
\mathbf{f}^{z} \\
\mathbf{f}^{y}
\end{array}\right] .
\end{aligned}
$$

The only source of damping is the control strategy in the AMBs. The lateral vibrations in $y$ - and $z$-directions are coupled by gyroscopic effects of the rigid disks. For safety reasons, retainer bearings acting at discrete positions along the rotary shaft are applied but these are not in the focus of the present study. The operational deflection of the present rotor is assumed to be sufficiently small such that rotor-stator contacts are excluded and the linearisation of the electromagnetic force in Eq. (7) remains valid.

A time-periodic stiffness variation in the rotor system is realised in the AMBs by introducing a time-dependent proportional action $k_{P}(t)$ in the PID controllers. This control parameter is changed periodically for both AMBs simultaneously according to

$$
k_{P}(t)=k_{P}(1+\varepsilon \sin v t)
$$

resulting in the global time-periodic stiffness matrix

$$
\mathbf{C}(t)=\mathbf{C}_{0}+\varepsilon \mathbf{C}_{t} \sin v t
$$

where $\mathbf{C}_{0}, \mathbf{C}_{t}$ are time-independent coefficient matrices. 


\section{Numerical study}

The flexible rotor system in Fig. 1 is examined for a fixed set of system parameters listed in Table 1 for different values of the amplitude $\varepsilon$ and the control frequency $v$ of the timeperiodicity introduced in Eq. (15). The equations of motion in Eq. (13) are solved by direct numerical integration. Initially, the rotor shaft in Fig. 1 rests at the centre position $\mathbf{q}=\mathbf{0}$. Since unbalance forces $\mathbf{f}$ acts on the rotor system, the rotor shaft is deflected from this initial condition to a new deflection that rotates with the rotor speed $\Omega$. The transition between these two states is described by free vibrations. A sample time history for the radial deflection of the disk D2

$$
\left|r_{D 2}\right|=\sqrt{y_{D 2}^{2}+z_{D 2}^{2}}
$$

at constant, nominal AMB characteristics $(\varepsilon=0$ in Eq. (15)) and a constant rotor speed of $\Omega=601 / \mathrm{s}$ is shown in Fig. 2. The same features are observed for the time histories at the other disk positions and are therefore omitted. The first four natural frequencies obtained from

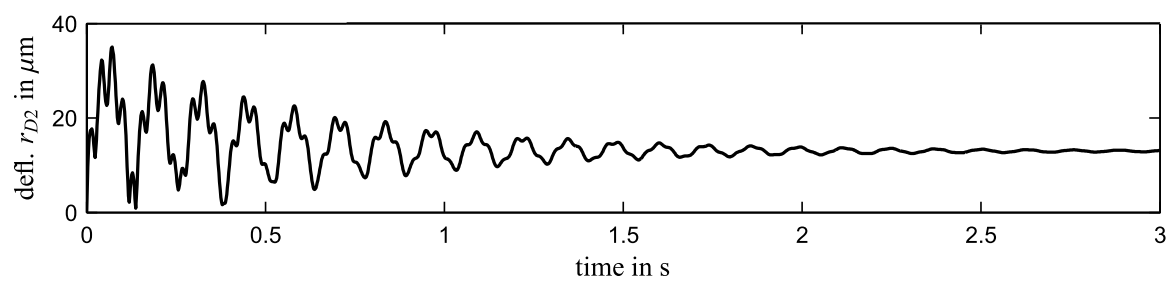

Fig. 2 Rotor deflection of disk D2 under the action of unbalance forces for $\varepsilon=0$.

an eigenvalue analysis of the undamped system in Eq. (13) at rest together with the resulting parametric resonance and anti-resonance frequencies are listed in Table 2. Evaluating the analytical predictions in ${ }^{(3)}$ reveals that for the present system only the parametric excitation frequencies of difference type in Eq. (1), $\left|\omega_{k}-\omega_{l}\right| / n$, correspond to parametric anti-resonance frequencies at which an increase of effective damping is achievable. Within the present investigation, the influence of gyroscopic effects on the first four natural frequencies is below $4 \%$. Note that the time history in Fig. 2 describes the radial deflection of a disk in a coordinate system that is fixed to the disk. Consequently, the observed frequency components correspond to the speed-dependent natural frequencies $\omega_{i}[\Omega]$ that are modulated by the rotor speed $\Omega$. The lowest frequency component in Fig. 2 becomes $\left|\Omega-\omega_{1}\right| / 2 \pi \approx 8 \mathrm{~Hz}$.

Table 1 Details of rotor system in Fig. 1.

\begin{tabular}{lll}
\hline & bending stiffness of rotor shaft & $41.4 \mathrm{~N} / \mathrm{m}$ \\
& mass per unit length of shaft & $0.4 \mathrm{~kg} / \mathrm{m}$ \\
rotor & total length of rotor and shaft diameter & $680 \mathrm{~mm}, 8 \mathrm{~mm}$ \\
& mass and axial moment of inertia of disk D1 & $0.78 \mathrm{~kg}, 0.4 \cdot 10^{-3} \mathrm{~kg} \mathrm{~m}^{2}$ \\
& mass and axial moment of inertia of disk D2 and D3 & $1.20 \mathrm{~kg}, 1.4 \cdot 10^{-3} \mathrm{~kg} \mathrm{~m}^{2}$ \\
& mass of studs in AMB1 and AMB2 & $0.88 \mathrm{~kg}$ \\
\hline \multirow{4}{*}{ AMBs } & number of electromagnets & 4 in each bearing \\
& magnetic bearing constant & $3.64 \cdot 10^{-6} \mathrm{Vsm} / \mathrm{A}$ \\
& current-force constant $k_{i}$ & $42.1 \mathrm{~N} / \mathrm{A}$ \\
& bearing stiffness $k_{s}$ & $1.052 \cdot 10^{5} \mathrm{~N} / \mathrm{m}$ \\
& controller sampling frequency & $8 \mathrm{kHz}$ \\
& radial clearance & $0.80 \mathrm{~mm}$ \\
\hline
\end{tabular}

Table 2 First few natural and parametric frequencies of the rotor system at rest, $\Omega=0$.

\begin{tabular}{|c|c|c|c|}
\hline \multicolumn{4}{|c|}{ natural frequencies } \\
\hline$\omega_{1}=110.61 / \mathrm{s}$, & $151.61 / \mathrm{s}$, & $\omega_{3}=265.01 / \mathrm{s}$, & $\omega_{4}=402.11 / \mathrm{s}$ \\
\hline \multicolumn{4}{|c|}{ main parametric resonance frequencies } \\
\hline $\begin{array}{l}2 \omega_{1}=221.21 / \mathrm{s} \\
\omega_{2}=262.21 / \mathrm{s},\end{array}$ & \multicolumn{2}{|c|}{$\begin{array}{c}2 \omega_{2}=303.21 / \mathrm{s} \\
\omega_{1}+\omega_{3}=375.61 / \mathrm{s}\end{array}$} & $\begin{array}{l}2 \omega_{3}=530.01 / \mathrm{s} \\
\quad \omega_{2}+\omega_{3}=416.61 / \mathrm{s}\end{array}$ \\
\hline \multicolumn{4}{|c|}{ main parametric anti-resonance frequencies } \\
\hline$\left|\omega_{1}-\omega_{2}\right|=41.01 / \mathrm{s}$ & $\left|\omega_{2}-\omega_{3}\right|=$ & $13.41 / \mathrm{s}, \quad \mid \omega_{3}$ & $\omega_{4} \mid=137.11 / \mathrm{s}$ \\
\hline
\end{tabular}


Now, the periodic open-loop control in the AMBs is switched on following the control law in Eq. (15). First, the vibration behaviour is investigated at a control amplitude of $\varepsilon=0.15$ and a fixed control frequency $v$ in the range between 0 and $4001 / \mathrm{s}$. Numerical integration of the equations of motion in Eq. (13) at $v=01 / \mathrm{s}$ results in the time history already shown in Fig. 2. All time histories in the frequency range of interest are summarised in the contour plot in Fig. 3. Light areas depict low values and dark areas high values of the disk deflection $\left|r_{D 2}\right|$. Additionally, frequency lines of the frequencies $v_{0}$ defined in Eq. (1) are plotted for the orders $n=1$ up to $n=5$ on the right hand side of the figure. Their line thickness is scaled by the order $n$. The frequencies listed in Table 2 are of order $n=1$ and are plotted as lines with the largest thickness. All possible frequency combinations $v_{0}$ are divided into three groups corresponding to the three block on the right hand side of the figure: the two groups $2 \omega_{i} / n$ and $\left(\omega_{k}+\omega_{l}\right) / n$ that correspond to destabilising parametric excitation frequencies, and the group $\left|\omega_{k}-\omega_{l}\right| / n$ which corresponds to stabilising parametric frequencies. These frequency lines help encoding the complex distribution of the time series. At each of these frequencies a dense frequency interval exist within which the system vibrations are either excited or damped. If these frequency intervals overlap, it depends which effect dominates. In general, the destabilising effect at frequencies $2 \omega_{i} / n$ dominates the effects acting at frequencies $\left|\omega_{k} \mp \omega_{l}\right| / n$.
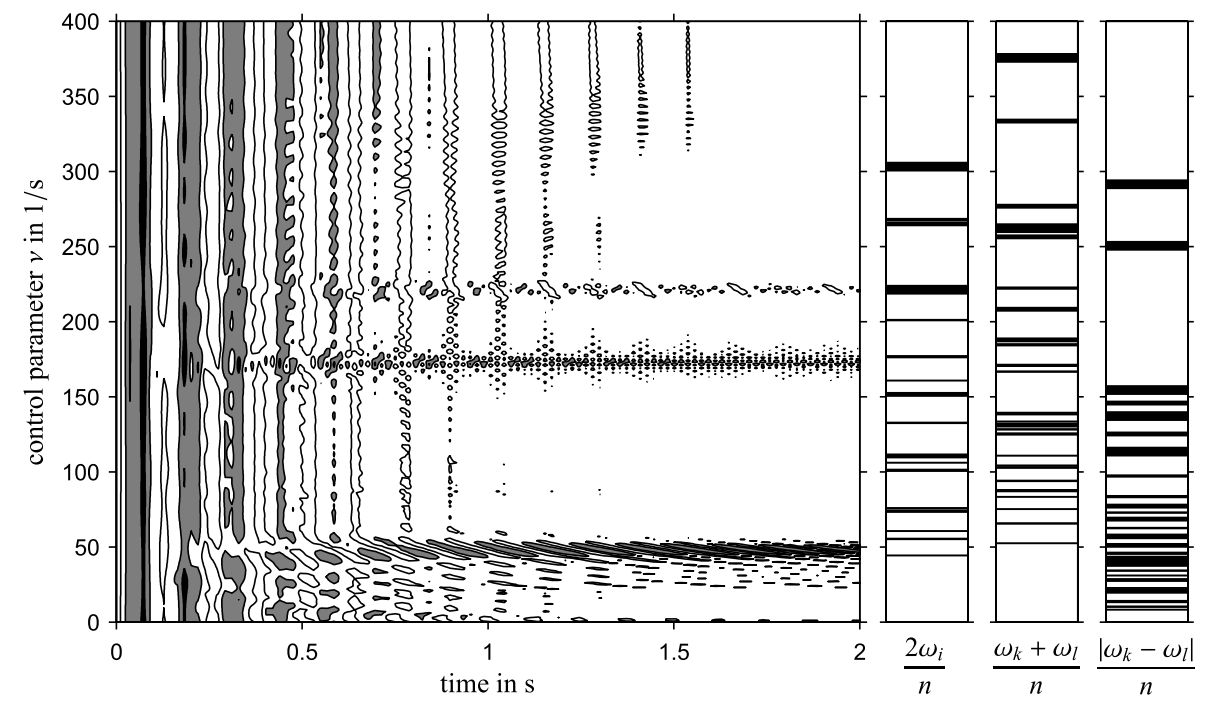

Fig. 3 Time histories of $r_{D 2}$ in dependency of the control parameter $v$ at $\varepsilon=0.15$.

Destabilising effects, a decrease in effective damping, are found where the control parameter $v$ is in the vicinity of the frequencies $491 / \mathrm{s}, 1721 / \mathrm{s}$ and $2211 / \mathrm{s}$. The corresponding parametric resonance frequencies can be identified by comparison with the frequency lines on the right hand side, e.g. the shaded area at $2211 / \mathrm{s}$ corresponds to the frequency $2 \omega_{1}=221.21 / \mathrm{s}$. Indentations in the distribution towards lower time values in Fig. 3 give hints of an increase in effective damping. These indentations can be found close to the frequencies $81 / \mathrm{s}, 731 / \mathrm{s}$, $1301 / \mathrm{s}$ and $2851 / \mathrm{s}$.

Figure 4 shows the time histories of the deflection $r_{D 2}$ at an increased control amplitude $\varepsilon=0.30$. A similar distribution as in Fig. 3 is encountered with the difference that already existing destabilising regions are enlarged and new emerge. For example, the narrow instability region is increased at the frequency $851 / \mathrm{s}$. In Fig. 3, an increase in the effective damping is generated due to a parametric anti-resonance frequency $\left|\omega_{k}-\omega_{l}\right| / n$ at $v=3001 / \mathrm{s}$. Increasing the control amplitude as in Fig. 4 destroys this stabilising effect and the destabilising frequency $2 \omega_{i}$ determines the global system behaviour. As mentioned previously, this is a common feature of this control strategy in case different frequency intervals overlap.

Figure 5 visualises the time histories after an increase of the control amplitude up to $\varepsilon=$ 


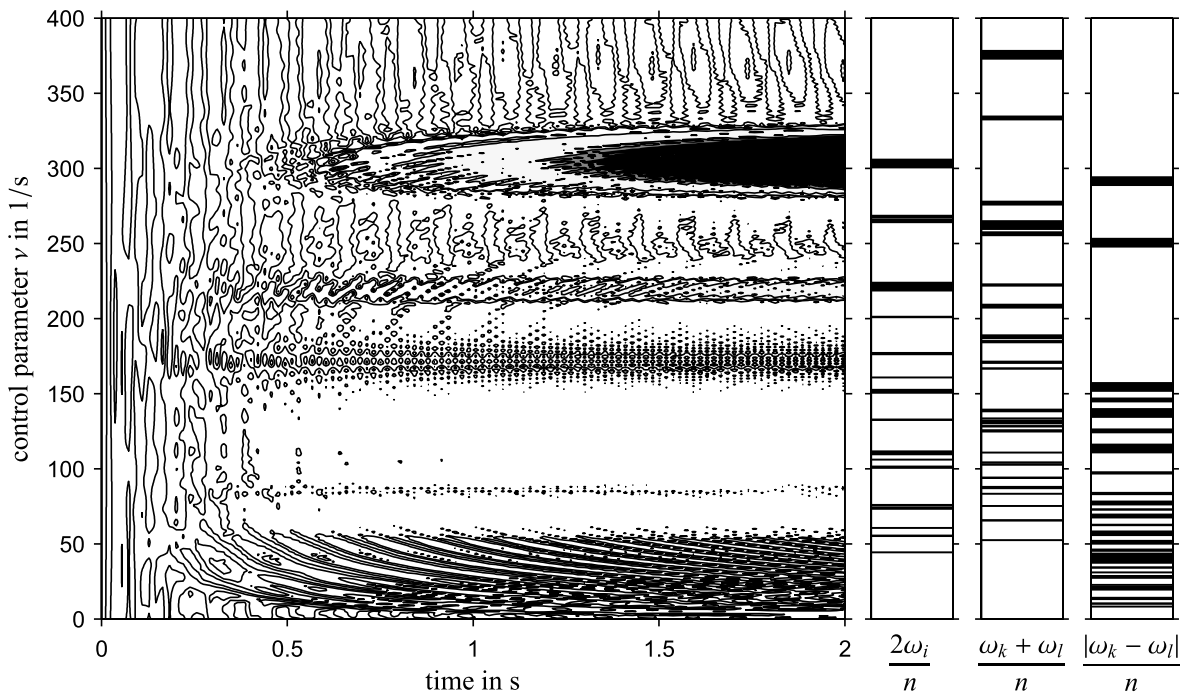

Fig. 4 Time histories of $r_{D 2}$ in dependency of the control parameter $v$ at $\varepsilon=0.30$.
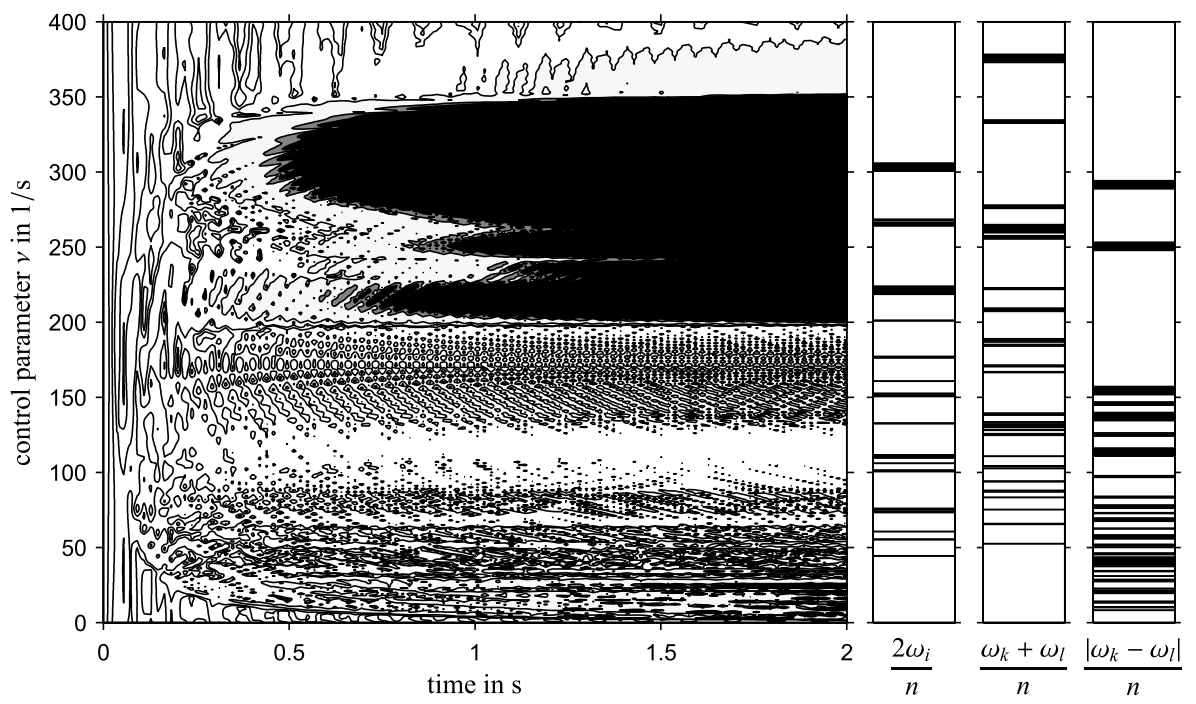

Fig. 5 Time histories of $r_{D 2}$ in dependency of the control paramter $v$ at $\varepsilon=0.45$.

0.45. At this high control amplitude several destabilising regions become effective, especially in the frequency range between 200 and 3501 /s and most of the stabilising control frequencies vanish. However, the main stabilising control frequency close to $v=\left|\omega_{2}-\omega_{3}\right|=113.41 / \mathrm{s}$ is still effective, even for this high control amplitude. This is the optimum control frequency to be chosen for the proposed open-loop control in Eq. (15) for this specific rotor system.

Table 3 Logarithmic decrement at the optimum control parameter $v=\left|\omega_{2}-\omega_{3}\right|$.

\begin{tabular}{ccc}
\hline control frequency $v$ in $1 / \mathrm{s}$ & control amplitude $\varepsilon$ & logarithmic decrement \\
113 & 0.00 & 1.9 \\
113 & 0.15 & 2.5 \\
113 & 0.30 & 3.9 \\
113 & 0.45 & 4.6 \\
\hline
\end{tabular}

The corresponding time histories of the deflection $r_{D 2}$ of disk 2 at the optimum control frequency $v=\left|\omega_{2}-\omega_{3}\right|$ at different values of the control amplitude $\varepsilon$ are shown in Figs. 6-8. The time history of the rotor system for switched off open-loop control $(\varepsilon=0)$ was presented in Fig. 2. Introducing the open-loop control in Eq. (15) at a moderate control amplitude of 
$\varepsilon=0.15$, increases the effective system damping slightly, see Fig. 6. A further increase of the controller amplitude increases the effective system damping further, see Figs. 7 and 8. Evaluating the logarithmic decrement of these time histories gives a good measure of the effective damping present in the rotor system. The determined values are summarised in Table 3. Hence, for the present system configuration the effective damping can be enhanced, or amplified, by a factor of 2.4 .

It has to be highlighted that this amplification factor of the effective damping depends strongly on the initial system damping. It was shown theoretically that the smaller the effective damping of the nominal system is, the higher the amplification factor becomes ${ }^{(6)}$. For example, decreasing the derivative action $k_{D}$ of the PID controller by a factor of 2, decreases the active damping $d_{m}$ in Eq. (10) by the same factor but doubles the amplification factor achievable by the open-loop control in Eq. (15).

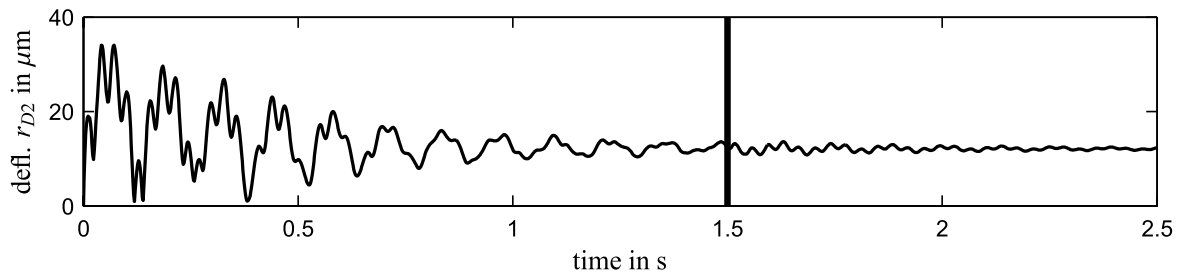

Fig. 6 Time history at optimal controller parameters $v=\left|\omega_{2}-\omega_{3}\right|$ and $\varepsilon=0.15$.

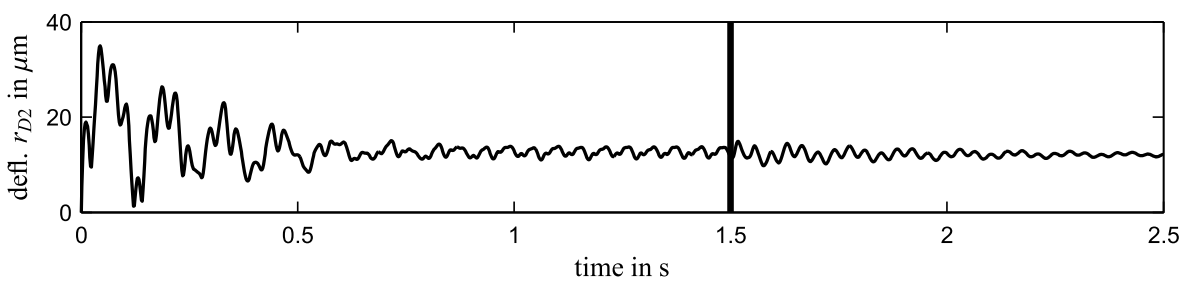

Fig. 7 Time history at optimal controller parameters $v=\left|\omega_{2}-\omega_{3}\right|$ and $\varepsilon=0.30$.

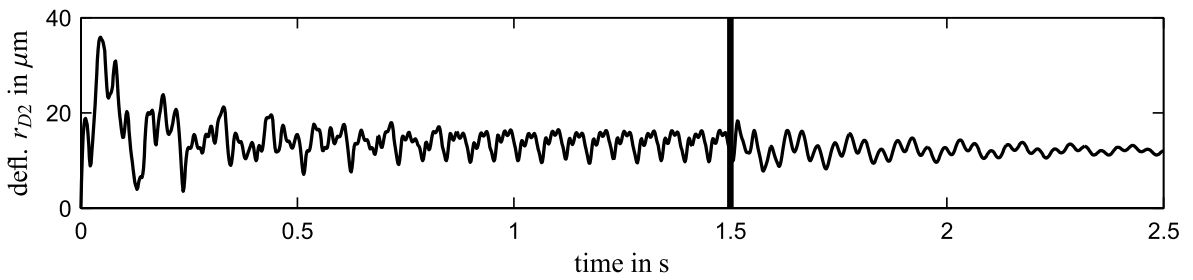

Fig. 8 Time history at optimal controller parameters $v=\left|\omega_{2}-\omega_{3}\right|$ and $\varepsilon=0.45$.

A comparison of Figs. 6-8 shows a characteristic feature of the proposed open-loop control method. A certain level of the control amplitude $\varepsilon$ has to be exceeded to achieve the damping effect for which the existing damping in the system is increased. Upon exceeding this value, an additional artificial damping is acting in the rotor system which increases the overall system damping. The drawback of the open-loop method applied to this example system is that although the transient vibration can be reduced significantly, a certain level of oscillations remains at the final state due to the persistent alteration of the bearing stiffness. These oscillation increase for higher values of the controller amplitude. It depends on the application whether these oscillations interfere with the desired operation task or the artificial increase in damping can be justified. A proper countermeasure could be a simple on/off logic that activates the open-loop control on demand only, e.g. when the radial deflection exceeds a predefined limit. The effect of a simple on/off switch is shown in Figs. 6-8. The proposed open-loop control is switched off at $1.5 \mathrm{~s}$. Hence, the artificially increased system damping is switched back to its initial value and the rotor vibrations decrease with the lower initial system damping induced by the inherent active damping in the AMBs. A soft on/off switching is suggested to avoid sudden changes in the stiffness characteristics leading to small but impact-like excitation. 


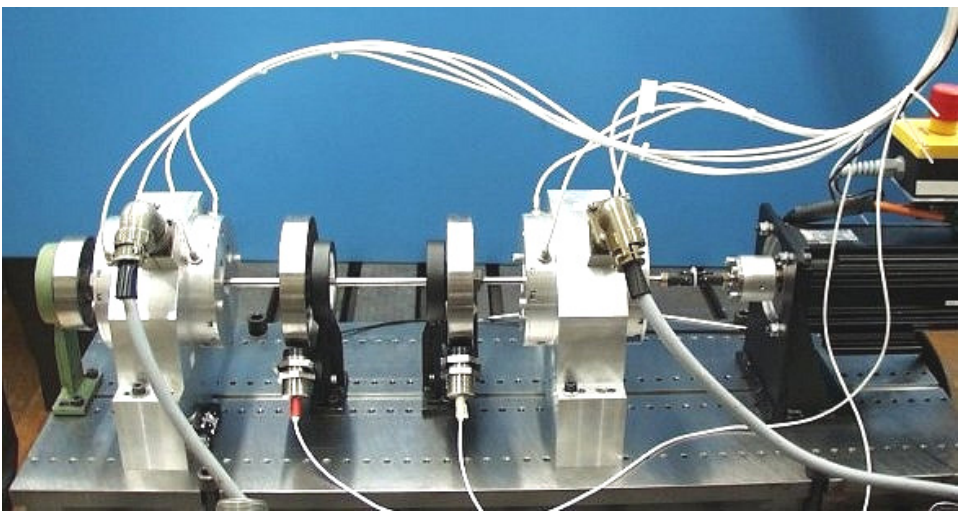

Fig. 9 Flexible rotor with multiple disks supported by two magnetic bearings.

\section{Experiment}

In this section experimental results are presented that underline the theoretical findings from above. Figure 9 shows the experimental realisation of the system introduced in Fig. 1 and Table 1: a flexible rotor system with multiple rigid disks which is supported by two AMBs. The electromagnetic forces generated in the AMBs depend on the rotor deflection and the magnetic field. The magnetic field can be changed in an arbitrary manner by the current provided to the electromagnets. The actual position of the rotor shaft is measured by inductive sensors (two for each direction $y$ and $z$ ). These signals are processed by the realtime controller hardware dSpace, which implements the PID controllers in Eq. (8) to regulate the currents provided to each of the electromagnets. In parallel to this PID control, an openloop control of the proportional action is implemented according to Eq. (15), which realises a periodic change in the active bearing stiffness according to Eq. (10).

At the sample rotor speed of $600 \mathrm{rpm}$ (below the first critical speed), the rotor is levitated in the AMBs by the PID controllers. Additionally, an open-loop control varies the proportional action $k_{P}$ at the optimum control frequency $v=\left|\omega_{2}-\omega_{3}\right|=113.41 / \mathrm{s}$ that was found in the previous theoretical section. At the time of $8 \mathrm{~s}$ an impulse is applied to the rotor system realised by adding an impulse-like current to the controller current. Time histories for different values of the control amplitude $\varepsilon$ are shown in Fig. 10. For the system without open-loop control $(\varepsilon=0)$, the system response decays with an effective logarithmic decrement of 0.8 . Activating the open-loop control at the optimum frequency enhances the system damping artificially which results in increased logarithmic decrements. A value of 1.6 is achieved for the logarithmic decrement at a control amplitude of $\varepsilon=0.15$ and a value of 3.7 at $\varepsilon=0.30$. Higher control amplitudes are not possible with the present set-up of the rotor test rig.

Without the proposed open-loop control $(\varepsilon=0)$, the system damping can be increased

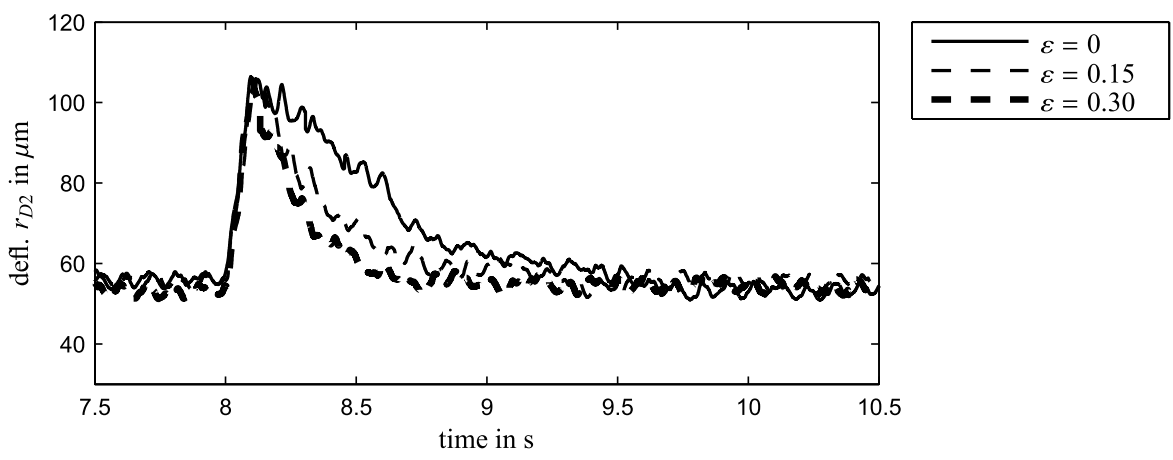

Fig. 10 Measured time histories at the optimal controller parameter $v=\left|\omega_{2}-\omega_{3}\right|$. 
simply by the active damping $d_{m}$ in Eq. (10) introduced by the PID controllers, too. However, this increase is limited by the level of measurement noise in the control loop and its amplification by the derivative action $k_{D}$. Starting from optimum PID controllers in the nominal system, $k_{D}$ can be amplified maximally by a factor of 1.7 . Introducing the proposed open-loop control at the control parameters $\varepsilon=0.3$ and $v=\left|\omega_{2}-\omega_{3}\right|$, allows an increase in effective system damping well above this factor. The maximum amplification factor for the effective damping that is achievable experimentally is 4.6 which lies well above the limit realisable by conventional active damping.

\section{Conclusions}

In this study new findings on damping by parametric excitation are presented. A flexible rotor system is analysed having support bearings with time-periodic, open-loop control of the stiffness coefficients. It is verified theoretically and demonstrated experimentally that the proposed open-loop control method is capable to increase the overall damping of a flexible rotor system. The open-loop method possesses several advantages. First, introducing a periodic change in the bearing stiffness works as an open-loop system with no feedback control necessary. Secondly, the control method can be applied in parallel with existent methods, since it affects mainly the free vibrations of a system. Finally, in the present system, the proposed control method is capable of increasing the effective damping well above limitations due to measurement noise.

\section{References}

( 1 ) Bolotin, V.V., Dynamic stability of elastic systems, Holden-Day, 1964.

( 2 ) Cartmell, M., Introduction to linear, parametric and nonlinear vibrations, Chapman and Hall, 1990.

( 3 ) Dohnal, F., Damping by parametric stiffness excitation: resonance and anti-resonance, Journal of Vibration and Control 14, 2008.

( 4 ) Dohnal, F. and Mace B.R., Amplification of damping of a cantilever beam by parametric excitation, Proceedings of the 9th International Conference on Motion and Vibration Control, Munich, 2008.

( 5 ) Dohnal, F. and Mace B.R., Damping of a flexible rotor by time-periodic stiffness and damping variation, Proceedings of the 9th International Conference in Vibrations in Rotating Machinery, Oxford, UK, 2008.

( 6 ) Dohnal, F., Damping of mechanical vibrations by parametric excitation: parametric resonance and anti-resonance, Südwestdeutscher Verlag, 2009.

( 7 ) Gèrardin, M. and Rixen, D., Mechanical vibrations - Theory and application to structural dynamics, Wiley, 1994.

( 8 ) Nayfeh, A.H. and Mook, D.T., Nonlinear Oscillations, John Wiley and Sons, 1995.

( 9 ) Tondl, A., To the problem of quenching self-excited vibrations, Acta Technica CSAV 43, Institute of Electrical Engineering, Academy of Science Czech Republic, 1998.

(10) Tondl, A., Self-excited vibration quenching in a rotor system by means of parametric excitation, Acta Technica CSAV 45, Institute of Electrical Engineering, Academy of Science Czech Republic, 2000.

(11) Yamamoto, T. and Saito A., On the vibrations of summed and differential types under parametric excitation, Mem Faculty of Engng 22, Nagoya University, Japan, 1966.

(12) Yakubovich, V.A. and Staryhinskii, V.M., Linear differential equations with periodic coefficients, 1 and 2, Wiley, 1975. 